Neurosurg Focus 25 (1):E5, 2008

\title{
The neurosurgical treatment of addiction
}

\author{
*Bianca M. L. Stelten, M.Sc., ${ }^{1}$ Lieke H. M. Noblesse, M.D., ${ }^{1}$ Linda ACKermans, M.D., ${ }^{1}$ \\ Yasin Temel, M.D., Ph.D., ${ }^{1,2}$ AND Veerle Visser-Vandewalle, M.D., Ph.D. ${ }^{1,2}$ \\ ${ }^{1}$ Maastricht Institute for Neuromodulative Development, and ${ }^{2}$ School for Mental Health and \\ Neuroscience, Maastricht University and Medical Centre, Maastricht, The Netherlands
}

\begin{abstract}
Addiction or substance dependence is a psychiatric disorder that affects many individuals in the general population. Different theories concerning the neurobiological aspects of addiction have been proposed. Special attention has been paid to models concerning dysregulation of the reward circuit and the inhibitory control system within the cortico-basal ganglia-thalamocortical pathways. In the past, attempts have been made to treat patients suffering from addiction by performing psychosurgery. Lesions were created in specific brain regions that were believed to be dysfunctional in addiction. Procedures such as cingulotomy, hypothalamotomy, and resection of the substantia innominata and the nucleus accumbens have been described as a treatment for severe addictive disorders. Deep brain stimulation, a neurosurgical treatment that has been proven to be a safe alternative for lesions in the treatment of movement disorders, has more recently been proposed as treatments for severe psychiatric conditions such as treatment-refractory obsessive-compulsive disorder and depression. With the expanding knowledge of the neurobiology of addiction, deep brain stimulation could be a future option in the treatment arsenal of addiction. (DOI: 10.3171/FOC/2008/25/7/E5)
\end{abstract}

\section{KEY WORDS • addiction • cingulotomy - deep brain stimulation - hypothalamotomy \\ - nucleus accumbens • psychosurgery}

A DDICTION or substance dependence is a chronic relapsing disease characterized by a strong psychological and physical dependence, resulting in a withdrawal syndrome when use of the drug is stopped. ${ }^{27}$ Both the Diagnostic and Statistical Manual of Mental Disorders, 4 th edition ${ }^{1}$ and the International Classification of Diseases, 10th edition ${ }^{45}$ define substance dependence as a psychiatric condition. Their definitions are quite similar, with criteria for tolerance, withdrawal, continued use despite problems, and indicators of impaired control. ${ }^{16}$

Addiction affects many people in the general population. Smoking is globally reported in 1 of 3 individuals. ${ }^{2}$ The 1 year prevalence of alcohol dependence is $7 \%^{10}$ in the general population. The United Nations World Drug Report ${ }^{40}$ shows abuse of cannabis by $0.1-17.7 \%$ of the population in the world. Other drugs are abused by $0.1-3 \%$ of the global population.

During the past decades, many theories have been proposed concerning the origin of addiction and dependence behavior. ${ }^{43}$ Addiction begins in most cases with social sub-

Abbreviations used in this paper: DBS = deep brain stimulation; DDS = dopamine dysregulation syndrome; OCD = obsessive-compulsive disorder; PD = Parkinson disease.

*Ms. Stelten and Dr. Noblesse contributed equally to this study. stance taking, then moves to compulsive use and finally to dependence and withdrawal. ${ }^{27}$ Positron emission tomography studies have shown that, when an individual takes a psychoactive substance, dopamine is released in the nucleus accumbens, which causes "the high." 19 By an increased dopamine release in the nucleus accumbens, the inhibition of the output neurons is decreased, which causes activation of the reward system. This circuit consists of dopaminergic neurons that project from the ventral tegmental area to the ventral striatum (including nucleus accumbens), the amygdala and septal nuclei, and prefrontal and cingulate cortices. $^{24,30}$ The feeling of well-being produced by activation of the reward system can be seen as positive reinforcement. Negative reinforcement involves escaping from or avoiding withdrawal symptoms, which occur after cessation of the substance use. ${ }^{23,43} \mathrm{Koob}^{23}$ suggested that in addicted individuals both positive and negative reinforcement involve disruption of the reward system by repeated administration of the substance.

Besides activation of the reward system, substance selfadministration causes increased dopamine release in the amygdala, which in turn strengthens associations between the rewarding qualities of the substance and exteroceptive stimuli. This could explain why an environment with substance-associated stimuli causes the craving reported by addicted individuals. ${ }^{18,24}$ 
On top of these reinforcing actions of the abused substance, impairment of inhibitory control and decision making is reported; these are caused by a dysfunction of the dopaminergic neurotransmission system in the prefrontal cortex and the anterior cingulate gyrus..$^{13,19,24,30}$ This leads to a diminished inhibition of unconditioned and conditioned responses, probably also by dopaminergic activation of the nucleus accumbens. ${ }^{18,24}$ This may cause an inability to resist substance-abusing behavior in addicted individuals, even though they are explicitly aware of the negative consequences. . $^{13,19,24,30}$

In the literature, addiction is often compared to OCD, and OCD is thought to be caused by a dysfunction in this inhibitory system as well. In OCD, however, an overactivation of the orbitofrontal cortex and the anterior cingulate cortex is reported, in contrast to addiction. This could cause the excessive concerns in patients with OCD about the future consequences of their actions. ${ }^{30}$

The reported models for the neurobiological changes in addiction are the modern basis for extended research concerning agents that possibly act on these circuits. Over the last century, though, before the constitution of these models, research has also been conducted into the possible effects of brain surgery, or psychosurgery as it is called, for addiction. In psychosurgery, a specific region of the brain is ablated to treat several (intractable) psychiatric conditions, including mood disorders, schizophrenia, OCD, and other anxiety disorders. The rationale for this kind of treatment was based on the assumption that a psychiatric condition was the effect of a malfunction of a specific anatomical region. . $9,17,31,41^{2}$

With the expanding knowledge of the neurobiological theories of addiction, however, a new surgical treatment option, DBS, may arise. The DBS procedure was originally introduced for the treatment of movement disorders, ${ }^{5}$ but nowadays it is being studied as a possible treatment option for intractable states of neuropsychiatric conditions such as Tourette syndrome, ${ }^{38,42}$ OCD, ${ }^{14,29}$ and depression. ${ }^{33,37}$ With DBS, an electrical imbalance at the level of the corticobasal ganglia-thalamocortical loops is thought to be modulated, so that symptoms are reduced.

The purpose of this review is to describe the different psychosurgical procedures used for the treatment of addiction and to discuss the first reports on the treatment of substance-related addictive disorders by using DBS.

\section{Methods}

We searched MEDLINE for all articles concerning surgery for addiction, using the following search terms: "addiction" (in all its dimensions), "dependence behavior," "psychiatric disorders," "surgery," "ablative surgery," "lesions," "psychosurgery," "cingulotomy," "cingulumotomy," "hypothalamotomy," "nucleus accumbens," and "deep brain stimulation." The articles obtained in this way were searched for relevant references. Only articles concerning original research on human subjects and articles written in English and German were included.

\section{Results}

Thirteen articles on surgery for treatment of addiction met the inclusion criteria: 11 articles reported on the results of psychosurgery in addiction, and 2 case reports were published on DBS in patients with comorbid addictive disorders.

\section{Cingulotomy Studies}

In the past, cingulotomy, or "cingulumotomy" as it is described by some authors, was used for the treatment of various psychiatric illnesses, such as major affective disorders, chronic anxiety disorders, chronic pain, and OCD, with different results. ${ }^{4,6,26,32,41}$ In case of addiction, the aim of the procedure was to interrupt obsessional thoughts about drug use, by creating a lesion in the cingulate gyrus. ${ }^{34}$

In 1962, Foltz and White ${ }^{11}$ published the results of unilateral or bilateral stereotactic lesions in the anterior cingulum created by electrocoagulation to treat patients with chronic intractable pain. Over a period of 7 years, 16 patients with psychogenic pain and pain due to organic or neoplastic disease were included. Fourteen patients in this group were also addicted to narcotics. In 12 patients, excellent or good results were reported for pain relief. None of the addicted patients required narcotics after the operation, and only 5 showed mild signs of withdrawal in the first 48 hours postoperatively. In the remaining 9 patients, no signs of withdrawal were observed. No difference was seen between unilateral and bilateral cingulotomy. No details are given about the long-term effects of this operation in these patients.

In 1973, Balasubramaniam et al. ${ }^{3}$ analyzed the results of 28 addicted patients who were treated using bilateral stereotactic cingulotomy. It is one of the first studies in which addiction was the primary indication for cingulotomy. Most of these patients were addicted to pethidine or morphine, and the mean age was 41 years. All patients had an obsessive-compulsive desire to take drugs. Twenty-one patients had undergone previous psychiatric therapy without any effect. Treatment was considered a failure if the patients took at least 1 tablet, got at least 1 injection, or consumed alcohol at least 1 time postoperatively. None of the 28 treated patients reported any discomfort, and in 22 individuals the treatment was successful, with an immediate effect. The follow-up period ranged from 4 months to 2 years. Extreme self-confidence and euphoria were reported immediately after surgery in all but 2 patients. No longterm complications were described.

In 1974, Sharma ${ }^{39}$ reported a total and permanent abolition of opiate hunger following bilateral anterior cingulotomy for intractable pain. The 3 patients reviewed suffered from severe "obsessive-compulsive neuroses" and were addicted to opiates for 3-15 years. Surgery was performed by open craniotomy. After surgery, 1 patient showed flattening of affect, which resolved after 3 weeks. There was no opiate craving, and the patients showed no evidence of abstinence syndrome. No description was given about the patient characteristics and severity of addiction.

Four years later, Kanaka and Balasubramaniam ${ }^{20}$ analyzed the results in 73 addicted patients who were treated by lesions in the anterior cingulum. The operations took place between 1970 and 1976, and the patient age varied from 25 to 52 years. Of all the included patients, $75 \%$ had undergone psychotherapy preoperatively. Immediately after surgery none of the patients craved drugs, and in most 
of the patients withdrawal symptoms were absent. If the patient ingested drugs or alcohol at least once, the surgery was considered a failure. This treatment was successful in $80 \%$ of morphine addicts, $90 \%$ of meperidine addicts, and in $68 \%$ of alcohol addicts, with a follow-up duration varying from 1 to 6 years. Relapse occurred only within 6 months of surgery. This study showed no psychological or neurological deficits after surgery. Kanaka and Balasubramaniam proposed that the procedure could be a promising cure for drug addiction.

In 2003, Medvedev et al. ${ }^{34}$ investigated the long-term effects of bilateral cryocingulotomy in 348 patients with strong heroin dependence. Their dependence behavior was characterized by an obsessive-compulsive component. The duration of drug addiction ranged from 2 to 15 years. All patients had been repeatedly treated in "a medical institute with non-invasive methods" without effect. Examination of the patient's mental state was performed before and after surgery. In 4 patients, postoperative complications consisted of intracerebral and subdural hematoma or postoperative infections. At the moment of discharge, all patients reported absence of craving for drugs. After 2 years, $45 \%$ of the 187 patients interviewed had completely abstained from drugs and $17 \%$ were in remission for $\geq 2$ years after 1 or 2 instances of drug taking in the past. In $13 \%$ there was a partial improvement, and $12 \%$ showed no change. In $13 \%$ of the cases no data were available. Medvedev et al. proposed that the full effect of the surgery would not manifest itself immediately and concluded that the positive outcome in this study was $62 \%$, including the $17 \%$ of the patients who showed a single instance of drug taking postoperatively. Medvedev et al. also concluded that psychological dependence in drug addiction could be a manifestation of OCD. Effective treatment, though, should not just include treatment for OCD, but also for drug addiction.

The aforementioned studies have not reported any longterm behavioral changes. Lenhard et al., ${ }^{28}$ though, described the case of a 67-year-old woman who had developed disturbed oral impulse control and impaired, divided attention and executive function after unilateral anterior cingulotomy for therapy-resistant alcohol dependence. Nevertheless, her drinking behavior changed from chronic to episodic. Cohen et al. ${ }^{7}$ also described attention and executive impairments after cingulotomy performed for intractable pain. These impairments partially resolved after 12 months. The anterior cingulate cortex is involved in reward-based decision making, and it mediates motivational and attentional responses to drug cues, so cingulotomy could cause impairments in these functions. ${ }^{7,28}$

\section{Hypothalamotomy Studies}

In 1973, Müller et al. ${ }^{35}$ reported a case of alcohol addiction treated with unilateral ventromedial hypothalamotomy. The patient was a 30-year-old man, for whom previous treatments had been unsuccessful. The early effect seemed promising, but the patient showed a relapse into alcoholism during the follow-up period of 11 months. The authors suggested that this procedure could be successful if performed bilaterally.

Five years later, Dieckmann and Schneider ${ }^{8}$ published the results of hypothalamotomy in 15 patients with alcohol and drug addiction. The case report by Müller et al. ${ }^{35}$ and the animal experiments by Kerr and Pozuelo ${ }^{21}$ led to this study on stereotactic hypothalamotomy as a treatment for addiction in patients. Most of the patients suffered from polyvalent drug addiction and alcoholism, and all patients had been treated previously with abstinence in combination with other therapies. The follow-up duration ranged from 2 to 3 years. High-frequency lesions were placed in the ventromedial nucleus of the hypothalamus, unilaterally or bilaterally. Stereotactic hypothalamotomy seemed to help the patients regain their self-control. All patients showed a reduction in sexual drive. In those who underwent bilateral hypothalamotomy, 4 of 6 patients suffered from severe side effects: lack of impulse, amnestic syndrome, vision disorder, and vegetative crisis were reported. Because of the severe side effects, Dieckmann and Schneider concluded that the use of this surgical target in treating addiction should be limited until more research is done.

\section{Resection of the Substantia Innominata}

In 1969, Knight ${ }^{22}$ published the results of stereotactic surgery of the substantia innominata in drug addiction. He successfully treated a patient suffering from chronic anxiety, who was addicted to barbiturates and alcohol. Knight also used this procedure in 5 heroin addicts. Postoperative observations showed alleviation of withdrawal symptoms, which made it easier to wean the patients from drugs. Knight did not explain why he chose the substantia innominata as a target for treatment of addiction. No information was given about patient characteristics and no details were provided about the outcome.

\section{Ablation of the Nucleus Accumbens}

In 2003, Gao et al. ${ }^{12}$ reported on ablation of the nucleus accumbens to treat addiction, based on several animal experiments and recent studies that had revealed the relationship between psychological dependence induced by drug addiction and the mesocorticolimbic dopamine circuit. Ablation of the nucleus accumbens would lead to blockage of the mesocorticolimbic dopamine circuit. This would prevent craving for drugs after detoxification and in this way cause reduction in the relapse rate. Stereotactic surgery was performed in 28 patients who had been drug addicts for at least 3 years. Gao et al. created bilateral lesions in the core of the nucleus accumbens with electrodes. The mean follow-up period was 15 months. Complete remission was reported in 7 patients, whereas 10 patients relapsed within 6 months, but experienced alleviation of withdrawal symptoms. Two individuals had a poor outcome, meaning relapse within 6 months, without alleviation of withdrawal symptoms. Seven patients were not included in the analysis and 2 were lost to follow-up. In 2 cases personality was slightly changed, and 4 patients suffered from temporary memory loss, so the incidence rate of complications was $19.2 \%$. All patients recovered within 1 month, though. Gao et al. concluded that bilateral ablation of the nucleus accumbens is a safe procedure for treatment of addiction.

\section{Deep Brain Stimulation}

Deep brain stimulation as we know it today was introduced in 1987 as a treatment for tremor. ${ }^{5}$ Today it is widely used for movement disorders such as PD. Over the last 2 
decades it has also been investigated as a treatment for refractory psychiatric disorders such as Tourette syndrome $^{38,42}$ and OCD. ${ }^{14,29}$

Two case reports have been published about patients with comorbid addiction problems who underwent DBS as a treatment for other conditions. In 2005, Witjas et al. ${ }^{44}$ reported on 2 patients suffering from PD with severe DDS who underwent bilateral subthalamic nucleus DBS for dyskinesias and motor fluctuations. The DDS is characterized by severe dopamine addiction, which is caused by misregulation of the brain reward system. This results in loss of control over drug taking by the patient with PD.

The first patient in the report of Witjas et al. ${ }^{44}$ was a 38year-old man with an 8-year history of PD, with excessive alcohol intake and severe DDS. After surgery, dopaminergic treatment was stopped, and 2 years later he was still completely drug free. The patient had only 1 episode of compulsive alcohol intake, which occurred 6 months after surgery. The second patient was a 53-year-old man with a 5 -year history of PD who had severe DDS. Prior attempts to decrease his dopaminergic intake were unsuccessful. After surgery, dopamine treatment was reduced by $75 \%$. After 18 months, the patient only needed a low dose of dopaminergic medication. In both cases there was a good effect on motor disability, but also on DDS and related behavior characteristics. Motor improvement after surgery made it possible to stop or decrease the dopaminergic treatment, thereby causing an improvement in behavioral disorders. The authors suggested, though, that addiction could have been stopped because of a direct effect of DBS on the reward-seeking circuit in the brain.

Two years later, Kuhn et al..$^{25}$ published a case report of a 54-year-old patient with severe agoraphobia with panic attacks, secondary depressive disorder, and alcohol dependency. He was treated by bilateral DBS of the nucleus accumbens. After surgery the patient showed only a slight reduction of his anxiety disorder and depression, but he showed a remarkable change in his substance abuse. One year after surgery, the patient consumed alcohol only occasionally. The authors concluded that investigation of this incidental finding in future studies can confirm the role of the nucleus accumbens in addiction.

\section{Discussion}

Addiction is a psychiatric condition with many individual, social, and financial implications. Over the past century, different surgical procedures have been performed to relieve addiction. In this review we describe studies in which cingulotomy, hypothalamotomy, and ablation of the substantia innominata and the nucleus accumbens are performed.

The studies on cingulotomy show good results, but the authors differ in their definitions of success and failure and the conclusions concerning the clinical outcome. Medvedev et al. ${ }^{34}$ conclude that full treatment effects become visible only after a while, in contrast to Kanaka and Balasubramaniam ${ }^{20}$ and Balasubramaniam et al., ${ }^{3}$ who believe in an immediate effect. In these articles, the side effects occurring after cingulotomy are scarcely described. Lenhard et al. ${ }^{28}$ and Cohen et al. ${ }^{7}$ report on attention and executive impairments after cingulotomy, though.

Although hypothalamotomy has shown some effect on the addicted patient's ability to regain self-control, the severe side effects-especially in the bilateral procedureare reason not to use this technique in the treatment for substance addiction. ${ }^{8,35}$ The only article on ablation of the substantia innominata for addiction is not clear in its clinical outcome. ${ }^{22}$

The nucleus accumbens seems to be a more promising target for the relief of addiction. Nevertheless, besides the article of Gao et al., ${ }^{12}$ which shows a complete remission of the addictive symptomatology in more than a quarter of the patients, no other reports of ablation of the nucleus accumbens for drug addiction in patients have been published yet.

Caution is needed when interpreting the results of the articles on psychosurgery for the treatment of addiction. In a recent editorial ${ }^{15}$ and letter ${ }^{36}$ concern is expressed about the Russian ${ }^{34}$ and Chinese articles ${ }^{12}$ reviewed here. There were no randomized control groups during research, and it is not clear whether the surgically treated patients were able to give free and informed consent for the surgery because of the punitive policies in these countries toward drug abuse.

Another concern in psychosurgical treatment of addicted patients is the irreversibility of the lesion caused by the procedure. Deep brain stimulation, a neurosurgical technique used as a treatment for movement disorders, only causes a microlesion in the target structure, which disappears in the weeks following surgery. The influence of the local current by the stimulator is completely reversible, just by turning off the device. This reversibility creates the unique possibility for conducting a randomized, double-blind trial, because the patient is generally not able to discriminate the difference between stimulation on or off.

So far, only reports on the use of DBS in patients with comorbid addiction disorders have been published, ${ }^{25,44}$ so extended research has to be conducted to create more insight into the optimal target, the mechanism of action, and the procedure's safety regarding treatment in a psychiatric condition such as addiction. With the expanding knowledge of the neuronal circuits responsible for the behavioral disorders associated with addiction, though, DBS could become a future treatment option for patients suffering from addiction.

\section{Conclusions}

Addiction is a severe psychiatric disorder with a high individual and socioeconomic impact. In the past, several neurosurgical procedures have been described, with lesions being made in specific brain regions thought to be dysfunctional in addiction. Deep brain stimulation might be a safe alternative. More research is needed to understand better the underlying mechanism of addiction, and to define selection criteria for the patients who might benefit from this surgery.

\section{References}

1. American Psychiatric Association: DSM-IV-TR: Diagnostic and Statistical Manual of Mental Disorders, ed 4. Washington DC: American Psychiatric Association, 2000

2. Bala M, Strzeszynski L, Cahill K: Mass media interventions for smoking cessation in adults. Cochrane Database Syst Rev 1: CD004704, 2008 
3. Balasubramaniam V, Kanaka TS, Ramanujam PB: Stereotaxic cingulumotomy for drug addiction. Neurol India 21:63-66, 1973

4. Ballantine HT Jr, Bouckoms AJ, Thomas EK, Giriunas IE: Treatment of psychiatric illness by stereotactic cingulotomy. Biol Psychiatry 22:807-819, 1987

5. Benabid AL, Pollak P, Seigneuret E, Hoffmann D, Gay E, Perret J: Chronic VIM thalamic stimulation in Parkinson's disease, essential tremor and extra-pyramidal dyskinesias. Acta Neurochir Suppl (Wien) 58:39-44, 1993

6. Christmas D, Matthews K, Eljamel MS: Neurosurgery for mental disorder. Br J Psychiatry 185:173-174, 2004 (Letter)

7. Cohen RA, Kaplan RF, Moser DJ, Jenkins MA, Wilkinson H: Impairments of attention after cingulotomy. Neurology 53:819824, 1999

8. Dieckmann G, Schneider H: Influence of stereotactic hypothalamotomy on alcohol and drug addiction. Appl Neurophysiol 41:9398, 1978

9. Feldman RP, Goodrich JT: Psychosurgery: a historical overview. Neurosurgery 48:647-659, 2001

10. Ferri M, Amato L, Davoli M: Alcoholics Anonymous and other 12-step programmes for alcohol dependence. Cochrane Database Syst Rev 3: CD005032, 2006

11. Foltz EL, White LE Jr: Pain "relief" by frontal cingulumotomy. J Neurosurg 19:89-100, 1962

12. Gao G, Wang X, He S, Li W, Wang Q, Liang Q, et al: Clinical study for alleviating opiate drug psychological dependence by a method of ablating the nucleus accumbens with stereotactic surgery. Stereotact Funct Neurosurg 81:96-104, 2003

13. Goldstein RZ, Volkow ND: Drug addiction and its underlying neurobiological basis: neuroimaging evidence for the involvement of the frontal cortex. Am J Psychiatry 159:1642-1652, 2002

14. Greenberg BD, Malone DA, Friehs GM, Rezai AR, Kubu CS, Malloy PF, et al: Three-year outcomes in deep brain stimulation for highly resistant obsessive-compulsive disorder. Neuropsychopharmacology 31:2384-2393, 2006

15. Hall W: Stereotactic neurosurgical treatment of addiction: minimizing the chances of another 'great and desperate cure'. Addiction 101:1-3, 2006

16. Hasin D, Hatzenbuehler ML, Keyes K, Ogburn E: Substance use disorders: Diagnostic and Statistical Manual of Mental Disorders, fourth edition (DSM-IV) and International Classification of Diseases, tenth edition (ICD-10). Addiction 101 (1 Suppl):59-75, 2006

17. Heller AC, Amar AP, Liu CY, Apuzzo ML: Surgery of the mind and mood: a mosaic of issues in time and evolution. Neurosurgery 59:720-739, 2006

18. Jentsch JD, Taylor JR: Impulsivity resulting from frontostriatal dysfunction in drug abuse: implications for the control of behavior by reward-related stimuli. Psychopharmacology 146:373390, 1999

19. Kalivas PW, Volkow ND: The neural basis of addiction: a pathology of motivation and choice. Am J Psychiatry 162:1403-1413, 2005

20. Kanaka TS, Balasubramaniam V: Stereotactic cingulumotomy for drug addiction. Appl Neurophysiol 41:86-92, 1978

21. Kerr FW, Pozuelo J: Suppression of physical dependence and induction of hypersensitivity to morphine by stereotaxic hypothalamic lesions in addicted rats. A new theory of addiction. Mayo Clin Proc 46:653-665, 1971

22. Knight G: Chronic depression and drug addiction treated by stereotactic surgery. Nurs Times 65:583-586, 1969

23. Koob GF: The neurobiogy of addiction: a neuroadaptational view relevant for diagnosis. Addiction 101 (1 Suppl):23-30, 2006

24. Koob GF, Le Moal M: Neurobiology of Addiction. London: Academic Press, 2006

25. Kuhn J, Lenartz D, Huff W, Lee S, Koulousakis A, Klosterkoetter $\mathrm{J}$, et al: Remission of alcohol dependency following deep brain stimulation of the nucleus accumbens: valuable therapeutic impli- cations? J Neurol Neurosurg Psychiatry 78:1152-1153, 2007

26. Laitinen LV: Psychosurgery. Stereotact Funct Neurosurg 76:239-242, 2001

27. Le Moal M, Koob GF: Drug addiction: pathways to the disease and pathophysiological perspectives. Eur Neuropsychopharmacol 17:377-393, 2007

28. Lenhard T, Brassen S, Tost H, Braus DF: Long-term behavioral changes after unilateral stereotactic cingulotomy in a case of therapy-resistant alcohol dependence. World J Biol Psychiatry 6: 264-266, 2005

29. Lipsman N, Neimat JS, Lozano AM: Deep brain stimulation for treatment-refractory obsessive-compulsive disorder: the search for a valid target. Neurosurgery 61:1-13, 2007

30. Lubman DI, Yücel M, Pantelis C: Addiction, a condition of compulsive behavior? Neuroimaging and neuropsychological evidence of inhibitory dysregulation. Addiction 99:1491-1502, 2004

31. Malhi GS, Sachdev P: Novel physical treatments for the management of neuropsychiatric disorders. J Psychosom Res 53: 709-719, 2002

32. Mashour GA, Walker EE, Martuza RL: Psychosurgery: past, present, and future. Brain Res Brain Res Rev 48:409-419, 2005

33. Mayberg HS, Lozano AM, Voon V, McNeely HE, Seminowicz D, Hamani C, et al: Deep brain stimulation for treatment-resistant depression. Neuron 45:651-660, 2005

34. Medvedev SV, Anichkov AD, Polyakov YI: Physiological mechanisms of the effectiveness of bilateral stereotactic cingulotomy against strong psychological dependence in drug addicts. Human Physiology 29:492-497, 2003

35. Müller D, Roeder F, Orthner H: Further results of stereotaxis in the human hypothalamus in sexual deviations. First use of this operation in addiction to drugs. Neurochirurgia (Stuttg) 16:113-126, 1973

36. Orellana C: Controversy over brain surgery for heroin addiction in Russia. Lancet Neurol 1:333, 2002

37. Schlaepfer TE, Cohen MX, Frick C, Kosel M, Brodesser D, Axmacher N, et al: Deep brain stimulation to reward circuitry alleviates anhedonia in refractory major depression. Neuropsychopharmacology 33:368-377, 2008

38. Servello D, Porta M, Sassi M, Brambilla A, Robertson MM: Deep brain stimulation in 18 patients with severe Gilles de la Tourette syndrome refractory to treatment: the surgery and stimulation. J Neurol Neurosurg Psychiatry 79:136-142, 2007

39. Sharma T: Abolition of opiate hunger in humans following bilateral anterior cingulotomy. Tex Med 70:49-52, 1974

40. United Nations International Drug Control Programme: World Drug Report. Northamptonshire, UK: Oxford University Press, 2007

41. Valenstein ES: Great and Desperate Cures: the Rise and Decline of Psychosurgery and Other Radical Treatments for Mental Illness. New York: Basic Books, 1986

42. Visser-Vandewalle V, Ackermans L, van der Linden C, Temel Y, Tijssen MA, Schruers KR, et al: Deep brain stimulation in Gilles de la Tourette's syndrome. Neurosurgery 58:E590, 2006

43. West R: Theory of Addiction. Oxford: Blackwell Addiction Press, 2006

44. Witjas T, Baunez C, Henry JM, Delfini M, Regis J, Cherif AA, et al: Addiction in Parkinson's disease: impact of subthalamic nucleus deep brain stimulation. Mov Disord 20:1052-1055, 2005

45. World Health Organization: ICD-10: International Classification of Diseases. Lyons: World Health Organization, 1990

Manuscript submitted March 15, 2008.

Accepted April 15, 2008.

Sources of support: none reported.

Address correspondence to: Veerle Visser-Vandewalle, M.D., Ph.D., Maastricht Institute for Neuromodulative Development, University Medical Centre Maastricht, Oxfordlaan 10, 6229 EV Maastricht, The Netherlands. email: vva@snch.azm.nl. 